\title{
Assessment of AISI 431Grade Stainless Steel properties by Vacuum Tempering Process
}

\author{
Gandla Lakshmi Prasanna ${ }^{*}, J$ Saranya $^{2}$ and Ram Subbiah ${ }^{2}$ \\ ${ }^{1}$ Post Graduate student, Mechanical Department, Gokaraju Rangaraju Institute of Engineering and Technology, Hyderabad, India. \\ ${ }^{2}$ Associate Professors, Gokaraju Rangaraju Institute of Engineering and Technology, Hyderabad, India.
}

\begin{abstract}
The wear behaviour of AISI 431 stainless steel was examined under a dry sliding touch at compatible load with the vacuum tempering cycle. The method adopted was found to be effective in enhancing the wear conduct of stainless steel products, there by advancing the pliability quality. For the sample, vacuum tempering (VT) procedure for $\left(\mathrm{VT}_{1}\right) 2$ hours, $\left(\mathrm{VT}_{2}\right) 4$ hours and $\left(\mathrm{VT}_{3}\right) 6$ hours respectively, a low temperature heat procedure was performed. Untreated stainless steel was used as a kind of viewpoint tool for contrast with specific hardening samples and observed wear check. The hardening samples expose those moulded hard layers which improve the hardness of the surface. Predictions of small scale hardness showed a significant change in hardness during diagnosis. This operation improves the hardness of the material to up to 150 percent. For wear inspection, a pin on the disc drive is used to assess wear failure. The layers were observed by optical lens magnification and examination under electron magnifying lens.
\end{abstract}

\section{Introduction}

Resistance to corrosion and stainless steels, low maintenance, fairly inexpensive and natural lustre make it a suitable base material for a range of industrial applications. While these stainless steels make up a small percentage of the total amount of steel made, they are of technical and economic significance as they are the primary products for the oil, coal, process, and power industries. In many respects the impact of these alloy elements differs between hard-enable and non-hardenable stainless steels [6]. In salt water conditions, alloying compounds such as molybdenum improves resistance to pitting corrosion (Peckner and Bernstein 1977). Energy, like fuel, titanium, aluminium, and copper. Nickel, molybdenum, and nitrogen are used to modify its structure and strengthen properties such as resilience and durability in formability (Seriks John 1979). Untempered martensitic steels, though very hard are too weak to be useful in most applications [1]. Most applications include the quenched part tempering, affecting some hardness and further enhancing ductility. In order to arrive at an ideal heat treatment technique for a sensible combination of stiffness and tensile properties, recent research findings and study results of mechanical tests were carried out on various heat-medium carbon steel samples. [2-4] These samples were subjected to several heat treatment processes at various temperatures, consisting of full annealing, hardening, quenching and tempering of the oil. Samples treated with heat were then mechanically tested for the stiffness (Rockwell) and tensile properties (yield strength, total traction strength, ductility). It was demonstrated by mechanical analysis of medium carbon steel samples with rise in temperature. [5] Temperature effect on Mechanical properties of Medium Carbon Metal. Un-tempered martensitic steels, although very hard are too weak to be useful in most applications [8] Most applications include the quenched part tempering, impacting some durability and improving ductility [6]. In order to arrive at an ideal heat treatment technique for a sensible combination of toughness and tensile properties, recent research studies and analyses of mechanical test findings were carried out on various heattreated medium carbon steel samples $[10,11]$. Mechanical properties such as degree of hardness and tensile between three forms, lowest stiffness of the oil quenching sample and lowest elongation of the annealed sample. [12] This is how heat treatment plays a major role in the mechanical properties of modern steel and its corrosion resistance.

\section{Experimental procedure}

A vacuum tempering process is used to harden the 150 $\mathrm{mm}$ long, $10 \mathrm{~mm}$ thick disc, to its saturated limit. The pin value is length of $30 \mathrm{~mm}$ and diameter of $8 \mathrm{~mm}$, respectively. The specimens were machined and cut with wire cutting via electric discharge machine. The approach to study includes a laboratory procedure for testing clothing wear, utilizing a pin tool when sliding. Compounds are tested in groups, in nominally nonconditions. By using this wear measuring tool approach the main areas of experimental interest are identified [8] . The coefficient of friction is measured, too. On disc wear check, two specimens are used to conduct the device a pin and a disc. The disc is rotated against a moving pin that 
runs at a steady charge. Wear test shall be supplemented directly with untreated here with 6 conditioned samples. The pin and disc wear measurements are reported separately as volume loss in millimetres. The wear total is calculated by measuring all pre- and post-test specimens with the required linear measurements. Dimensional wear measurements are mostly used in practice, because loss is too small to measure precisely. While measuring mass loss the element of mass loss is converted into volume loss using a rational approximation for the specimen number. The example below demonstrates a design diagram of a typical pin-on-disc wear-check. The system here consists of a driven spindle and chuck to carry the spinning disc, a lever-arm device to keep the pin and adapters to press the pin specimen with a controlled pressure against the spinning disc specimen. The wear track on the disc is a circle, where several passes on the same path are received. The composition of AISI 431 Grade Stainless steel W.r.t A. Rajasekhar et al.[7] i.e. elements in percentage (\%) is carbon (C)-0.17, Manganese (Mn) -1, Silicon (Si) -0.6, Phosphorus (P)-0.04, Sulphur (S) -0.03, Chromium (Cr) 17, Nickel (Ni)-2 and Ferrous (Fe)-79.16.

The wear test experimental results of samples and weight loss in grams (gms) are obtained as follows untreated sample (UNT) - 0.0066, Vacuum Tempered $1\left(\mathrm{VT}_{1}\right)$ $0.0020 \mathrm{gms}$, Vacuum Tempered $2\left(\mathrm{VT}_{2}\right)-0.002 \mathrm{gms}$, Vacuum Tempered $3\left(\mathrm{VT}_{3}\right)-0.001 \mathrm{gms}$.

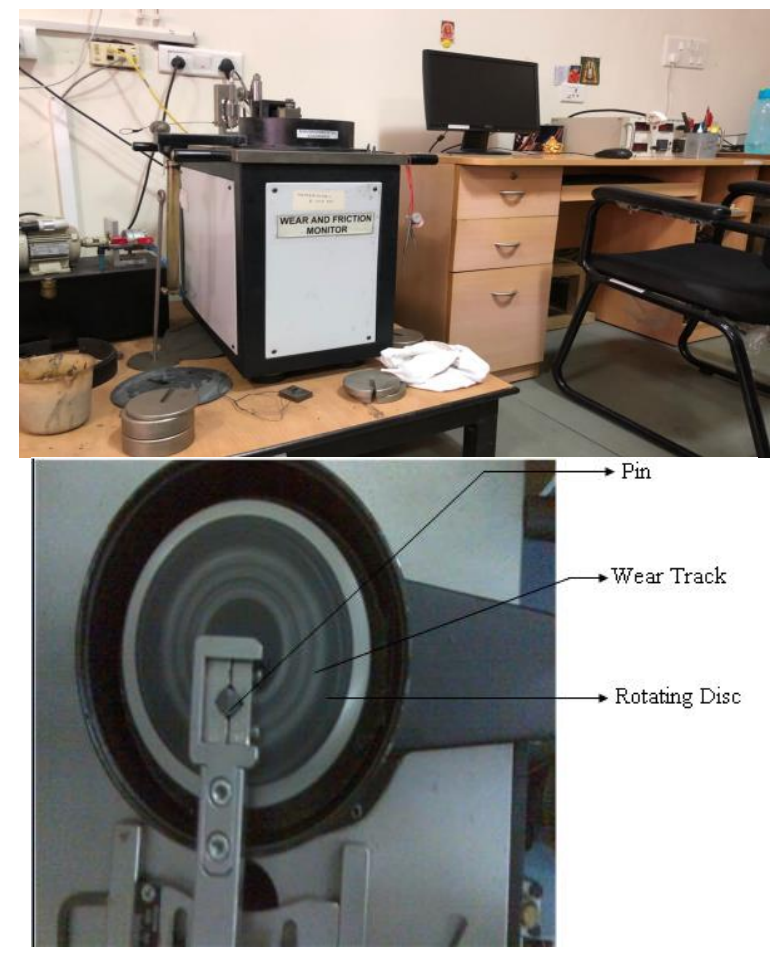

Fig 2.1 Wear Track obtained on Wear Testing machine @ BITS, Hyderabad

\section{Results and Discussion}

The specimens undergo reheat treatment process in the tempering method and the case depth was determined to be $\left(\mathrm{VT}_{1}\right) 11$ microns, $\left(\mathrm{VT}_{2} \& \mathrm{VT}_{3}\right) 12.5$ microns and
$\left(\mathrm{VT}_{4}\right) 15$ microns. In the tempering process, the case depth gets increased as the temperature timing increases. All ductility and toughness should be supported fairly in tempering processes. It should be noticed that the soil comprises a fairly smooth coating of ferrite and cement. The iron nitride development may be due to the discontinuous white areas found at the top of the hardened base.

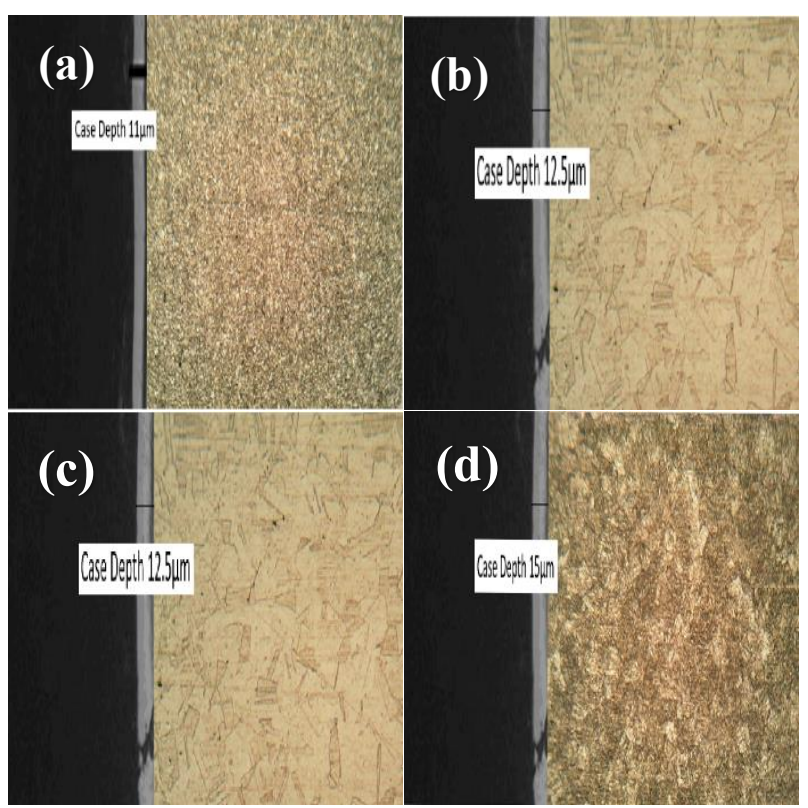

Fig. 3.1 Microstructure and case depths of (a. untreated (UNT)), (b. Vacuum Tempering 1(VT 1$)$ ), (c. Vacuum Tempering 2(VT2)), d. (Vacuum Tempering $3\left(\mathrm{VT}_{3}\right)$ )

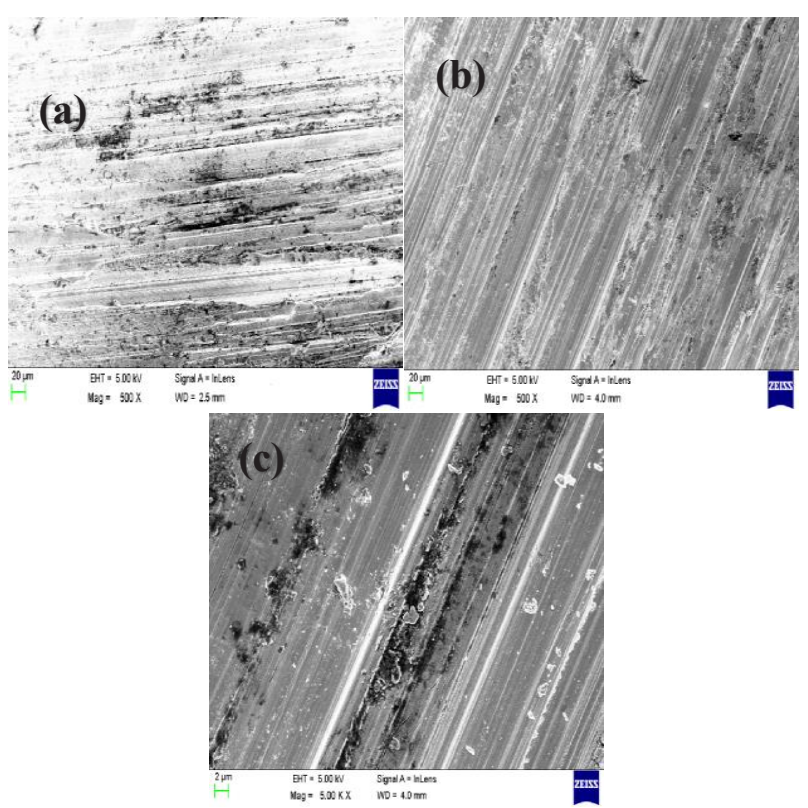

Fig. 3.2 SEM images of (a. Vacuum Tempering 1(VT1)), b. (Vacuum Tempering 2(VT2)), (c. Vacuum Tempering 3(VT $\left.\mathrm{VT}_{3}\right)$ )

From the findings of the scanning electron microscope, further material peeling from untreated specimen was found. As the tempering process period rises, corrosion declines and wear degradation decreases on the products made of stainless steel. There has been observed to be less plastic fatigue when it is prone to testing. This improves 
the wear strength of the material, increasing the ductility properties of stainless steel products and thus reducing the toughness.

\section{Conclusion}

The effect of the vacuum tempering treatment method on the wear action of martensitic stainless steel AISI 431 was tested using a pin on the disc tool and was ultimately examined by various metallographic measures. The key findings of the study are:

1. The modest oxidative wear mode was measured within the range of wear tracking control variables used. The key explanation for the reduction in wear rate was attributed to the reinforcement of martensite matrix by fine carbide precipitates.

2. In the tempering phase, strength and ductility were similarly stabilised due to the reheat treatment of stainless steel products.

\section{References}

1. S. Lu, K. Yao, Y. Chen, M. Wang, N. Chen, X. Ge, Metall. Mater. Trans. 46A (2015) 6090-6101.

2. G. Chakraborty, C.R. Das, S.K. Albert, A.K. Bhaduri, V. Thomas Paul, G. Panneerselvam, A. Dasgupta, Mater. Charact.100 (2015)81-87.

3. Baloji, D., Anil, K., Satyanarayana, K., Singh, S. K., \& Naik, M. T. Material Today 18, 4475-4481. (2019).

4. S.K. Bonagani, V. Bathula, V. Kain, Corrosion Science, Volume 131, Pages 340-354. (2018).

5. B. Sunil Kumar, V. Kain, A. Singh, Material Science and Engineering, Volume 700, Pages 140151.(2017)

6. Tummala Suresh Kumar, Kosaraju Satyanarayana, Materials Today: Proceeding, 26(2), 3228-3233 (2020)

7. A.Rajasekhar, G.Madhusudhan Reddy, T. Mohandas, V.S.R. Murti, Volume 30,Pages 16121624.(2009)

8. G. Sha, A. Morley, A. Cerezo, G.D.W. Smith, Philos. Mag. Lett. 87 (2007) 327-339.

9. Manikonda, R. D., Kosaraju, S., Raj, K. A., \& Sateesh, N., Material Today 5, 20104-20109. (2018)

10. T.J. Mesquita, E. Chauveau, M. Mantel, N. Bouvier, D. Koschel, Corros. Sci. 81 (2014) 152-161.

11. A.N. Isfahany, H. Saghafian, G. Borhani, J. Alloys Comp. 509 (2011) 3931-3936.

12. J. Chen, V. Radmilovic, T.M. Devine, Corros. Sci. 30 (1990) 477-494. 